\title{
Mujeres y emprendimiento a través de Lean In: una perspectiva crítica ${ }^{1}$
}

\author{
Maria Medina-Vicent ${ }^{2}$
}

Recibido: 01 de septiembre de 2017 / Aceptado: 19 de febrero de 2018

Resumen. La lógica del emprendimiento empresarial responde a los intereses del neoliberalismo y requiere de la construcción de los individuos como sujetos autoresponsables, autoexigentes y despolitizados para su expansión. En este contexto de mercantilización de las capacidades humanas, la posición social de las mujeres se vuelve más que vulnerable. Dicha constitución de las mujeres como sujetos emprendedores debe enmarcarse en la confluencia entre neoliberalismo y género, donde se crea el espacio para una peligrosa alianza: el feminismo neoliberal. A través de dicha lógica las mujeres son construidas como sujetos neoliberales, cuestión que permite a su vez la cabida del emprendimiento como nueva lógica de vida. En el presente trabajo se establecerá el marco teórico del fenómeno acabado de mencionar, identificando el discurso del emprendimiento en la literatura gerencial, particularmente la dirigida a mujeres a través del análisis del discurso del bestseller titulado Lean In de Sheryl Sandberg (2013).

Palabras clave: neoliberalismo; emprendimiento; feminismo neoliberal; literatura popular del management.

\section{[en] Women and Entrepreneurship Through Lean In: A Critical Perspective}

Abstract. The logic of entrepreneurship responds to the neoliberal interests and requires the construction of individuals as self-responsible, self-demanding and depoliticized subjects for its expansion. In this context of the commercialization of human capacities, the social position of women becomes more than vulnerable. The constitution of women as entrepreneurs should be framed on the confluence between neoliberalism and gender, where is the space to reflect on a dangerous alliance: the neoliberal feminism. It is through this logic that women are built as ideal neoliberal subjects, an issue that allows the entrepreneurship discourse as a logic of women's life. In the present work the theoretical framework of the phenomenon mentioned will be established, identifying the discourse of entrepreneurship in popular management literature, particularly that addressed to women, through the analysis of the bestseller Lean In by Sheryl Sandberg (2013).

Key words: neoliberalism; entrepreneurship; neoliberal feminism; popular management literature.

Sumario: 1. Introducción. 1.1. Objetivos. 1.2. Metodología. 2. La influencia del neoliberalismo en el movimiento feminista. 2.1. La subjetividad emprendedora en las mujeres. 3. Lean In: emprender para retroceder en la lucha feminista. 3.1. Abrazar el riesgo. 3.2. La despolitización de las mujeres. Conclusiones. Referencias bibliográficas.

1 Este estudio se inscribe en el Proyecto de Investigación Científica y Desarrollo Tecnológico FFI2016-76753C2-2-P financiado por el Ministerio Español de Economía y Competitividad.

2 Universitat Jaume I. Departament de Filosofia i Sociologia

medinam@uji.es 
Cómo citar: Medina-Vicent, M. (2018) "Mujeres y emprendimiento a través de Lean In: una perspectiva crítica". Cuadernos de Relaciones Laborales, 36(2), 305-323.

\section{Introducción}

La teoría del entreployee fue desarrollada por los autores Hans J. Pongratz y Günter VoB (2003) para referirse a un tipo de trabajador que autoemprende e identificar un momento de transformación fundamental en la disposición de la sociedad sobre la capacidad de trabajo. Se trata de un sujeto que personifica las consecuencias de la individualización del trabajo de forma extrema, y que tiene como valores principales la autonomía y la autoresponsabilidad a la hora de decidir cómo organizar su tiempo y trabajo en unas condiciones sociales de gran precariedad. En la escena neoliberal, este modelo de trabajador tiene tres elementos centrales. En primer lugar, el incremento de la auto-responsabilidad que lleva a nuevas formas de autogobierno. Es decir, ahora los trabajadores no están tanto sujetos a la gestión de otros como a la suya propia. En segundo lugar, existe la tendencia a economizar el trabajo, esto quiere decir que los trabajadores individuales deben pensar constantemente en la eficiencia, ya que deben venderse a ellos mismos en el mercado. En tercer y último lugar, la vida privada suele manejarse como si de una organización se tratase, ya que los individuos precisan planificar hasta el último milímetro sus vidas para satisfacer las cambiantes necesidades del mercado, dando lugar a una extrema autoexplotación de sus capacidades y su tiempo (Bröckling, 2016).

Dichas cuestiones se enmarcan en lo que el autor Richard Sennett ha dado en llamar "nuevo capitalismo" (Sennett, 2000; 2006; 2009), un escenario donde el individuo adquiere la responsabilidad de los riesgos que caracterizan al mercado, siendo partícipe del incremento de su propia precariedad (Alonso, Fernández Rodríguez y Rojo, 2016; Muñoz-Rodríguez y Santos-Ortega, 2017; Serrano y Martín, 2017). Cabe señalar que estos procesos de precariedad característicos del neoliberalismo se perpetúan a través de la difusión de los nuevos discursos del management (Fernández Rodríguez y Medina-Vicent, 2017), cuya herramienta de difusión más consumida es la literatura popular del management ( Jackson, 2002; Huczynski, 2006; Fernández Rodríguez, 2007). Este tipo de obras contienen un conjunto de ideas presentes en el pensamiento económico contemporáneo y sirven de guía para la actuación del/la directivo/a de la organización empresarial moderna, público al que se dirigen de forma directa (Clark y Salaman, 1996).

A través de esta literatura se consigue la difusión del espíritu del capitalismo (Boltanski y Chiapello, 2002), que sería la ideología que justifica el compromiso de las personas con el capitalismo, y que convierte este compromiso en una idea atractiva. En este sentido, el carácter predominante de la literatura managerial sería más ideológico que científico (Gantman, 2005; 2017). Una muestra de esta idea es el creciente carácter ficticio que caracteriza a los relatos contenidos en dichas obras (Lischinsky, 2008) y el surgimiento de toda una escuela, los Critical Management Studies, que tienen por objeto realizar una aproximación crítica a estos contenidos (Fernández Rodríguez, 2017). 
Cabe señalar pues, que este tipo de obras contribuyen a la fundamentación de lo que Christian Laval y Pierre Dardot denominan el "neosujeto" (2013), cuya subjetividad está íntegramente relacionada con las nuevas técnicas de "la empresa de sí", que consiguen que el individuo asuma la responsabilidad total ante los fracasos que le esperan en su camino hacia la supervivencia dentro de un mercado feroz. Se trata de una lógica mediante la que cada sujeto inicia el proceso de autogobernarse en base a un ethos empresarial donde el longlife training y la empleabilidad son bases centrales. De este modo, se consigue un gobierno empresarial basado en la responsabilidad individual y el autocontrol, premisas también divulgadas a través de la literatura managerial, que bailan al ritmo de las modas de gestión presentes en cada época (Collins, 2000: 20).

En este contexto de marcado neoliberalismo y precarización de las condiciones laborales, las mujeres se convierten en sujetos neoliberales ideales (Scharff, 2016b). En este caso, encontramos una subcategoría o subgénero dentro de la literatura popular del management dirigida expresamente al público femenino y surgido a raíz de la consolidación de la presencia de mujeres en los cargos de dirección. También en este caso, dicha literatura funciona como una herramienta de propaganda ideológica para la conformación de las mujeres como "neosujetos" neoliberales determinados por la lógica de la oferta y la demanda. Como señala la autora Elisabeth Kelan en referencia a las mujeres en el mundo del trabajo, las mujeres profesionales también participan de la lógica neoliberal: "most constructed themselves in terms of market-driven neoliberal subjectivities and accepted insecurity as part of their job and saw self-renewal as a way to preserve their market value" (Kelan, 2009: 143).

Por esta razón consideramos que acercarnos a la lectura crítica desde la perspectiva feminista (Reverter-Bañón, 2003) de la literatura popular del management dirigida a mujeres es un modo adecuado de identificar cómo se construye la lógica del emprendimiento a través de la subjetividad de las mujeres. Así pues, en el presente artículo de investigación reflexionaremos en torno a cómo se configura la identidad de las mujeres como sujetos emprendedores, es decir, qué lógicas argumentales y valores se utilizan para su construcción. Para esto, deberemos enmarcar su situación en la confluencia del feminismo con el neoliberalismo, algo que nos llevará a hablar del Neoliberal Feminism. Una vez identificadas las características o los discursos centrales de la construcción de la mujer como sujeto emprendedor, vernos cómo se reflejan dichos discursos en una de las obras más vendidas de los últimos años, esto es, Lean In de la directiva de Facebook Sheryl Sandberg. A través de este análisis del discurso seremos capaces de identificar los principales valores y discursos que subyacen a la constitución de las mujeres como emprendedoras y líderes de sus propias "vidas".

\subsection{Objetivos}

Los objetivos que se pretenden conseguir con la realización de esta investigación son dos. En primer lugar, enmarcar en qué contexto adquiere sentido la construcción de las mujeres como sujetos emprendedores y qué discursos confluyen para conformar dicho sujeto. Esto es, se hace necesario reflexionar en torno a la imbricación del neoliberalismo con el feminismo y la conformación de las mujeres como sujetos neoliberales. En segundo lugar, y una vez realizada dicha operación, se identificarán 
las bases argumentales y los valores sobre los que se construye el discurso sobre las mujeres emprendedoras. Para identificar dichos componentes se realizará un análisis del discurso de un bestseller de la literatura popular del management dirigida a mujeres de los últimos años (Lean In, de Sheryl Sandberg). Dicha literatura supone un vehículo de difusión masivo para las modas de gestión empresarial (Huczynski, 2006) y por tanto, reflejan la ideología propia del nuevo espíritu del capitalismo (Boltanski y Chiapello, 2002; Chiapello y Fairclough, 2002) también referida a la presencia de mujeres en la empresa (Kelan, 2008).

\subsection{Metodología}

La metodología empleada en el presente trabajo de investigación ha sido de carácter cualitativo y ha consistido en el análisis de contenido de la obra Lean In, perteneciente a un corpus mayor conformado por más de 50 obras. La selección de Lean In se ha realizado después de hacer una lectura aproximativa del corpus total en base a distintos criterios que se mencionan a continuación. En primer lugar, un criterio referido a las ventas, es decir, se ha tenido en cuenta el número total de ventas de la obra a nivel mundial. En segundo lugar, un criterio cronológico referido a que la obra hubiera sido editado entre los años 2007-2017, para poder identificar las últimas tendencias en este sentido. En tercer lugar, un criterio referido a la popularidad y el reconocimiento de las autoras en el mundo empresarial. En cuarto y último lugar, la repercusión mediática de la obra. Así pues, el gran número de ventas y la gran repercusión que ha tenido Lean In (Stewart, 2013; Fresier, 2014; Taylor, 2016) tanto en el mundo empresarial como en otros espacios, son rasgos suficientes para que nos decidamos a estudiar sus contenidos de forma crítica. Cabe recordar que el análisis de contenido se basa en la lectura (textual o visual) como instrumento de recogida de información, una lectura que debe realizarse siguiendo una sistematización (Alonso, 2003; Bryman, 2012).

\section{La influencia del neoliberalismo en el movimiento feminista}

Al igual que el discurso del emprendimiento en general encuentra su sentido en el marco del mercado neoliberal, el discurso específico del emprendimiento femenino encuentra su sentido en un contexto concreto: la influencia del neoliberalismo en el movimiento feminista. Así pues, si entendemos en una línea de pensamiento foucaultiana (Foucault, 2007), que el neoliberalismo no es solamente un sistema de estructuración mundial o económico, sino que es una forma de gobierno, y sobre todo un modo de gobernarse a uno/a mismo/a (Barry, Osbourne y Rose, 1996), nos resultará más sencillo comprender que éste influye en la construcción de las subjetividades de los sujetos.

Es decir, la lógica neoliberal y sus exigencias, tanto explícitas como implícitas, inciden radicalmente en los modos en que los/as ciudadanos/as se relacionan, construyen sus vidas y a sí mismos/as. De este modo, la concepción de las mujeres como emprendedoras promovida por la literatura popular del management dirigida a mujeres (Alwill, 2015; Huffington, 2007, 2015; Kay y Shipman, 2014; Kozmetsky, 1992; Mercadé, 1998; 2007), al influir en la conformación de las subjetividades 
femeninas, también responderá a ciertas exigencias neoliberales que tienen como objeto construir una subjetividad femenina de cierto tipo que sirva a los intereses de dicho sistema.

En este sentido, cabe preguntarse sobre la relación entre feminismo y neoliberalismo a la hora de configurar las subjetividades femeninas. Chaterine Rottenberg $(2013,2014,2017)$ afirma la existencia de lo que da en llamar Neoliberal Feminism, concepto que se refiere a la emergencia de ciertos discursos neoliberales dentro de la construcción de los sujetos feministas, es decir, novedades argumentales que nunca se habían dado a lo largo de la historia del feminismo y que resignifican a dicho movimiento, despolitizándolo y despojándolo de su carácter crítico. Mediante el análisis de las obras Why Women Still Can't Have It All (Slaughter, 2012) y Lean In (Sandberg, 2013), Rottenberg (2013) pretende identificar cuál es el marco feminista que alimenta los consejos dirigidos a mujeres que se pueden encontrar en estas dos obras.

En primer lugar, cabe señalar que la autora inscribe sus reflexiones en un marco territorial concreto, esto es, el de los Estados Unidos de América. Dentro de este prisma, señala un desplazamiento del Liberal Feminism al Neoliberal Feminism. Más concretamente, la autora propone que el Mainstream Liberal Feminism que predominaba en EEUU hasta hace pocos años y se exportaba al resto del mundo, está siendo desarticulado y transmutado en un modelo particular de gobernabilidad neoliberal (Rottenberg, 2013: 2). Este nuevo feminismo estaría en completa concordancia con el orden neoliberal, e incluso contribuiría a su desarrollo y exportación ${ }^{3}$.

Así pues, la clave central del Neoliberal Feminism se encuentra en que no ofrece ninguna crítica al neoliberalismo, sino más bien al contrario, se centra en construir una alianza con él. De este modo, se encarga de edificar un nuevo tipo de sujeto feminista: aquel que es consciente de la existencia de las desigualdades de género que perviven en nuestra sociedad; pero que acepta la responsabilidad individual total de su resolución, así como de su propio bienestar y cuidado, algo que estará específicamente relacionado con conseguir la felicidad y la satisfacción a través de lo que Rottenberg ha dado por llamar el modelo de la Balanced Woman (Rottenberg, 2013: 3; 2017: 331), una mujer que articula su presencia en el ámbito público y privado a través del equilibrio.

Este modelo de una mujer en equilibrio supone que la mujer individual es la que gestiona las contradicciones y obstáculos de su presencia en ambas esferas dicotómicas. Por tanto, estamos hablando de unos sujetos feministas caracterizados por el individualismo y la búsqueda de la felicidad particular, ideas que desarticulan por completo las bases colectivas de la lucha feminista que buscan la consecución de la justicia social. De este modo, tal y como Rottenberg indica: "The neoliberal feminist subject is thus mobilized to convert continued gender inequality from a structural problem into an individual affair" (Rottenberg 2013: 3). Y aquí es donde se encuentra la verdadera clave, ya que a través de esta lógica discursiva se puede comprender mejor la construcción de las mujeres como emprendedoras.

En los últimos años la filósofa Nancy Fraser ha denunciado de forma reiterada cómo el feminismo ha derivado en una mera "criada del capitalismo" (Fraser 2014). En este sentido, Fraser (2015) lanza una llamada a la recuperación del radicalismo en el movimiento feminista actual. Una llamada más que necesaria, si nos atenemos a la realidad que se desprende del Neoliberal Feminism. Mediante su trabajo nos recuerda que las sucesivas crisis que está sufriendo el régimen neoliberal, agudizadas por la globalización, amenazan la igualdad de forma multidimensional y que, en este escenario, las mujeres ocupan una posición crítica entre patriarcado y neoliberalismo. 


\subsection{La subjetividad emprendedora en las mujeres}

En esta sección veremos cómo la constitución del sujeto feminista neoliberal está estrechamente ligada con la lógica del emprendimiento. Trataremos de identificar los principales valores y argumentos discursivos de los que se nutre la lógica del emprendimiento a la hora de dirigirse a las mujeres. Nos centraremos pues, en la subjetividad femenina mainstream, esto es, la que coincide con el público al que se dirige la literatura popular del management dirigida a mujeres: mujeres blancas occidentales con estudios y carreras profesionales de éxito; que excluye los condicionantes de raza y clase (Wilson, 2011; 2012; 2015) y ofrecen una visión simplista del sujeto político del feminismo. En este sentido, resultará vital abordar la obra de Christina Scharff, quien identifica a las mujeres occidentales como sujetos "ideales" del neoliberalismo y estudia los procesos discursivos mediante los cuales conforman sus identidades emprendedoras (Scharff, 2016a: 217).

A partir de un análisis sociológico de grupos de mujeres profesionales en Alemania y Reino Unido, Scharff sostiene que la subjetividad femenina está economizada bajo el neoliberalismo, realidad que implica la construcción de las mujeres como emprendedoras de sí mismas. En esta construcción identitaria, las mujeres hablan de sí mismas como negocios que hay que gestionar, tal y como señala la autora: "entrepreneurial subjects relate to themselves as if they were a business, are active, embrace risks, capably manage difficulties and hide injuries" (Scharff, 2016c: 108). Por tanto, existen ciertos valores clave en la conformación de las emprendedoras: la proactividad, la ausencia de miedo a tomar riesgos, la capacidad de hacer frente a dificultades de diversa índole y la ocultación de vulnerabilidades y dependencias que las pudieran hacer parecer débiles o defectuosas en un contexto de marcada competitividad. A su vez, Scharff subraya la necesidad que tienen dichas mujeres de distanciarse de sí mismas para poder así tratarse como productos en constante mejora, es decir, concebirse como empresas (Gershon, 2011; Scharff, 2016c: 112).

Una de las consecuencias clave que se derivan de la organización de la sociedad en torno a una multiplicidad de empresas individuales es la despolitización de las relaciones políticas y sociales (McNay, 2009: 66). Dicha despolitización se produce por la propia lógica individualista y economicista que adquiere el sujeto como lógica de vida. Al situar al individuo por encima del grupo, se está acabando con los lazos que atan a las comunidades, y se está conformando un escenario en el que cada cual mirará solamente por sus propios intereses, se sustituye así el "nosotros/as" por el "Yo".

Scharff también ha identificado dicha cuestión en sus investigaciones, al percatarse de que las participantes en sus investigaciones no reflexionaban sobre las causas estructurales de sus problemas laborales o de otra índole: "there was no scope for politicization (...). Indeed, anger, as an affect that can give rise to politicization, was remarkebly absent from the research participants' accounts" (Scharff, 2016a: 221). Lo que ocurre normalmente es que dichas problemáticas se abordan como si se tratase de una cuestión esencialmente individual, el problema es de la mujer individual y es ella la que tiene que encargarse de solucionarlo. En gran medida su capacidad para solucionar dichos problemas será la que al mismo tiempo le otorgue legitimidad a su estatus como empresa eficiente en un contexto mayor.

Sin embargo, en el caso de las mujeres la despolitización se entrecruza con otra realidad: las desigualdades de género. Es decir, si bien la despolitización de las re- 
laciones sociales y políticas es una realidad generalizada en el contexto neoliberal, dicha despolitización en el caso de las mujeres se traduce en gran medida en un rechazo del feminismo o en su instrumentalización (Medina-Vicent, 2018a). En los discursos de estas mujeres son raramente traídas a colación los problemas y los obstáculos a los que tienen que hacer frente por cuestiones relacionadas con el género (Gill y Scharff, 2011). Las desigualdades por tanto, no pasan a formar parte de los discursos de los agentes. Se podría decir, tal y como apunta la autora Rosalind Gill, que el hecho de evitar hacer referencia a dichas desigualdades de género es parte de la construcción de la subjetividad neoliberal (Gill, 2014), que instrumentaliza la palabra "feminismo" y la convierte en un concepto vacío y totalmente innecesario a ojos de las mujeres más jóvenes.

En resumen, una de las cuestiones principales que hemos podido observar a través del trabajo de Scharff es que las mujeres como sujetos neoliberales repudian su vulnerabilidad y se acercan sin reflexionar a la toma de riesgos frente a las exigencias que les vienen del entorno neoliberal, algo que les convierte en más vulnerables si cabe. Desde este marco, se repudiará todo aquello que las haga ser concebidas como sujetos vulnerables, como, por ejemplo, el hecho de considerarse feministas. $Y$ es que, tal y como señala Scharff, el feminismo no se integra como un valor clave de la identidad neoliberal de las mujeres, porque es incompatible con un sujeto autosuficiente (Scharff, 2016b: 59). Dicho repudio del feminismo puede darse de forma directa, pero también es cierto que en muchas ocasiones responde más a una relación ambivalente que a un rechazo total. Esto explica en gran medida el carácter despolitizante del discurso del Neoliberal Feminism que encontramos en la literatura popular del management, donde se intuye una apuesta por la igualdad de género, pero no se menciona la palabra feminismo. Es decir, se habla de la necesidad de realizar pequeños cambios, pero siempre se evita instar a grandes transformaciones sociales.

Así pues, nuestra hipótesis inicial es que la literatura popular del management dirigida a mujeres también contribuye a la conformación de este tipo de subjetividad, algo que trataremos de identificar mediante el análisis de la obra Lean In. Por tanto, trataremos de identificar los enunciados que nos remitan a dos ideas centrales: la de la autogestión; y la de la negación de las dependencias y vulnerabilidades, que lleva irremediablemente a la despolitización del feminismo dentro de dicho discurso.

\section{Lean In: emprender para retroceder en la lucha feminista}

La autobiografía Lean In: Women, Work, and the Will to Lead (2013), de la actual CEO de Facebook, Sheryl Sandberg, aborda la experiencia personal y profesional de la autora, que al mismo tiempo aporta claves y consejos para el desarrollo profesional de las lectoras en el mundo del management. Además de hallarse en la lista Forbes The Most Powerful Women in Tech 2015, Sandberg ha conseguido un gran éxito mundial con esta obra (Pérez, 2013).

Dicha obra formaría parte de una subcategoría dentro de la literatura popular del management dirigida a mujeres, referida a biografías de mujeres ejecutivas y emprendedoras con éxito. Toda la obra se caracteriza por un tono políticamente correcto, que, aunque trata temas interesantes para el feminismo actual, lo hace de forma poco profunda y partiendo siempre de la experiencia individual de una mujer 
concreta. Teniendo en cuenta esta realidad y que la posición social de la autora se podría considerar como privilegiada dentro de las que otras mujeres ocupan alrededor del mundo, consideramos que dicha obra ofrece una visión del constructo "mujeres" demasiado homogénea.

Con una gran carrera profesional y gozando de gran prestigio en diferentes círculos empresariales, Sheryl Sandberg ha demostrado ser una persona de interés a la hora de hablar de las mujeres y la alta dirección empresarial (Sandberg y Grant, 2015), convirtiéndose en algo cercano a una gurú de la gestión. En este sentido, sus charlas abordan la presencia de las mujeres en dichas posiciones, y goza de gran popularidad en dichos seminarios y charlas alrededor del mundo (Sandberg, 2002). Además, la obra cuenta con su propia página web (http://leanin.org/book/), donde se ha inaugurado todo un círculo de experiencias de liderazgo de mujeres, donde los/ as visitantes pueden entrar y aportar sus propias vivencias, generando círculos de contactos. Partiendo del gran efecto que ha tenido la obra de Sandberg en la visibilización del tema de la igualdad de género en el mundo laboral, vamos a realizar a continuación un análisis del discurso con el objeto de encontrar las bases sobre las que se construye a las mujeres como sujetos emprendedores.

\subsection{Abrazar el riesgo}

Como hemos comentado anteriormente, la lógica de la autogestión y la negación de las dependencias y vulnerabilidades de las mujeres en el mercado laboral, que lleva irremediablemente a la despolitización del feminismo, son elementos centrales de la configuración de las mujeres como sujetos neoliberales emprendedores. A continuación, identificaremos los enunciados mediante los cuales se construyen dichas lógicas en la obra Lean In de la CEO Sheryl Sandberg. Al mismo tiempo, se detectarán los valores centrales de estos mismos discursos, que dan lugar a nuevas significaciones del modelo de género femenino.

En primer lugar, encontramos un valor central sobre el que se construye la subjetividad emprendedora: el riesgo. La idea de perder el miedo a tomar riesgos en el mundo de los negocios está más que consolidada. En el caso de las mujeres, el empujar a tomar riesgos supone reconstruir en cierta medida el estereotipo tradicional ligado a la feminidad inextricablemente asociado con el ámbito privado y las cuestiones de cuidado allí radicadas. Como se puede observar en los siguientes enunciados, se insta a las lectoras a tomar riesgos, a evitar el estancamiento y transformar su subjetividad para amoldarse a las exigencias de un mercado inestable que no acepta miedos ni vulnerabilidades:

But in business, being risk averse can result in stagnation (Sandberg, 2013: 61).

In many cases, women need to be more open to taking risks in (Sandberg, 2013: $61)$.

Esta lógica del riesgo viene aparejada de un valor concreto: la ambición. La toma de riesgos debe estar motivada por la ambición de llegar al éxito. Para integrar la ambición como un valor de las mujeres profesionales y emprendedoras, la autora debe primero desterrar una idea extendida en el imaginario social, esto es, 
la de que la ambición es un valor no asociado con el modelo de género femenino, históricamente relacionado con valores como la docilidad, la pasividad y la dependencia. Por tanto, el tratamiento que se desprende de dicho valor adquiere tintes de reconceptualización y de apertura a nuevos significados basados en las necesidades del neoliberalismo de que las mujeres también se gestionen a sí mismas como empresas exitosas:

Many women "still see ambition as a dirty word (Sandberg, 2013: 18).

Professional ambition is expected of men but is optional—or worse; sometimes even a negative-for women (Sandberg, 2013: 17).

I told the members of the graduating class that they should be ambitious not just in pursuing their dreams but in aspiring to become leaders in their fields (Sandberg, 2013: 25).

OKAY, so all a woman has to do is ignore society's expectations, be ambitious, sit at the table, work hard, and then it's smooth sailing all the way. What could possibly go wrong? (Sandberg, 2013: 39).

At this point, she probably scales her ambitions back even further since she no longer believes that she can get to the top (Sandberg, 2013: 94).

Reconceptualizar el valor de la ambición para que éste pueda ser asociado con las mujeres es una operación que se vuelve necesaria si se pretende que las mujeres emprendan en sus carreras y en sus propios negocios. Cabe remarcar que ésta es una línea argumental que da lugar a la creación de otras obras que se enmarcan dentro de la literatura popular del management, como por ejemplo Ambition is Not a Dirty Word de la autora Debra Condren (2006). Pero ¿de qué sirve la ambición si no tenemos un plan para rentabilizarla? El valor de la planificación y la autogestión de los proyectos de vida enfocada a la consecución del éxito empresarial y personal resultan evidentes en la obra de Sandberg:

We each have to chart our own unique course and define which goals fit our lives, values, and dreams (Sandberg, 2013: 10).

Just as I believe everyone should have a long-term dream, I also believe everyone should have an eighteen-month plan (Sandberg, 2013: 59).

But even a vague goal can provide direction, a far-off guidepost to move toward (Sandberg, 2013: 55).

En esta línea, aunque la planificación de la vida es un punto clave de la lógica neoliberal del emprendimiento, cuando abordamos la literatura popular de management dirigida a mujeres, encontramos una diferenciación en su formulación con respecto a la que realiza la literatura del management en general. Es decir, en el caso de la literatura dirigida expresamente a las mujeres profesionales y emprendedoras, la planificación de sus proyectos vitales implica prestar atención a la vida familiar, 
algo que no ocurre cuando la literatura adquiere una acepción más general y "neutra" respecto al género de su público:

But when it comes to integrating career and family, planning too far in advance can close doors rather than open them (Sandberg, 2013: 93).

I also know that all the planning in the world cannot prepare us for the constant challenges of parenting (Sandberg, 2013: 124).

For women who have achieved previous success by planning ahead and pushing themselves hard, this chaos can be difficult to accept (Sandberg, 2013: 125).

Dicho énfasis en la planificación familiar se deriva de la doble presencia de las mujeres en el ámbito público y el privado, es decir, a diferencia de los hombres, de los que no se esperan grandes actuaciones en la esfera de lo privado; las mujeres reciben presiones y expectativas de comportamiento desde ambas esferas. Esto implica que son las mujeres individuales las encargadas de gestionar la dicotomía entre ambos espacios $^{4}$, una dicotomía heredera de los principios de la filosofía moderna y los autores del contrato social/sexual (Pateman, 1995). Cuando no consiguen dar respuesta a dichas expectativas, se generan sentimientos de culpabilidad (Lewis, 2012) y la lógica de tener que escoger:

Because of work obligations, I've missed doctor's appointments and parent-teacher conferences and have had to travel when my kids were sick (Sandberg, 2013: 136).

Guilt management can be just as important as time management for mothers (Sandberg, 2013: 137).

Trying to do it all and expecting that it all can be done exactly right is a recipe for disappointment (Sandberg, 2013: 123).

En este sentido, Rottenberg señala que la construcción de las mujeres emprendedoras está directamente conectada con la idea de conseguir un feliz balance entre su vida laboral y familiar de forma individual (Rottenberg, 2013: 5). Se insta a las mujeres a que elaboren un plan de vida que les permita poder dar respuesta a sus "necesidades" de maternidad en el futuro. Se trata de inversiones o planificaciones que se realizan en el presente para ser amortizadas en el futuro (Rottenberg, 2017: 331-332), lo curioso es que en el caso de las mujeres dicha amortización se relaciona casi exclusivamente con el hecho de ser madres, casarse y conformar una familia (Lewis, 2010). Podemos observarlo en las siguientes citas:

$4 \quad$ El trabajo de Kristina A. Bourne y Marta B. Calás (2013) profundiza en los procesos discursivos mediante los cuales las mujeres emprendedoras establecen esta distinción entre "real work" y "not-real work" a la hora de configurar sus identidades como trabajadoras. Es decir, la radical distinción entre el tiempo que emplean en sus negocios y aquel tiempo que emplean en las tareas del hogar les sirve para construir su identidad laboral en función de si el trabajo que están realizando en cada momento será reconocido o no socialmente. 
The things that make the bad boys sexy do not make them good husbands (Sandberg, 2013: 115).

They told me that the most eligible women marry young to get a "good man" before they are all taken (Sandberg, 2013: 17).

I was twenty-four and convinced that marriage was the first -and necessary- step to a happy and productive life (Sandberg, 2013: 17).

Employed women reap rewards including greater financial security, more stable marriages, better health, and, in general, increased life satisfaction (Sandberg, 2013: 24).

When asked to choose between marriage and career, female college students are twice as likely to choose marriage as their male classmates (Sandberg, 2013: 92).

By the age of twenty-five, I had managed to get married ... and also divorced. At the time, this felt like a massive personal and public failure (Sandberg, 2013: 18).

Dicha futurición, que implica la toma de decisiones presente en función de los intereses futuros, contribuye a la conversión de las mujeres en capital humano listo para su explotación en cualquier momento, ya sea en un futuro más inmediato o más lejano. En base a los trabajos de Brown $(1995,2003,2016)$, Rottenberg subraya el papel de la futurición en la construcción de las mujeres como sujetos neoliberales a través de la idea del "reloj biológico" (Rottenberg, 2017: 339). Y es que, aquellas que deseen tenerlo todo (trabajo y familia), deberán atender a los límites temporales que marca ese supuesto reloj. Para Rottenberg, esta es una operación que resulta menos apremiante para los hombres, ya que su edad fértil, de algún modo, es más larga.

Si nos fijamos, lo que se consigue con esta operación es explotar las capacidades reproductivas de las mujeres, algo que también se hace a partir de la literatura popular del management, que, de forma general, da por sentado el deseo de las mujeres por tener familia convencional. Un ejemplo muy claro de esta cuestión lo vemos reflejado en la política de empresa de Facebook y Apple, que ofrecen a sus directivas la oportunidad de congelar sus óvulos (Jiménez Cano, 2014). Así se produce una completa mercantilización de la capacidad reproductiva de las mujeres, al tiempo que se convierte a la familia y a los futuros hijos/as en otra empresa más que gestionar (Rottenberg, 2013: 11). En el caso de las mujeres todas estas cuestiones se interconectan con el miedo a no responder a las exigencias sociales:

Fear is at the root of so many of the barriers that women face. Fear of not being liked. Fear of making the wrong choice. Fear of drawing negative attention. Fear of overreaching. Fear of being judged. Fear of failure. And the holy trinity of fear: the fear of being a bad mother/wife/daughter (Sandberg, 2013: 24).

En resumen, la toma de riesgos como eje central de la subjetividad emprendedora de las mujeres viene aparejada de la resignificación de valores como el riesgo y la ambición. Al mismo tiempo, se precisa de una planificación vital que permita a las mujeres asumir la gestión de su doble presencia en el ámbito público y privado, es decir, asumir la gestión individual de su doble explotación. El miedo a no poder dar 
respuesta a todo lo que se espera de estas mujeres profesionales funciona de forma bidireccional, y es precisamente dicho miedo el que es utilizado para seguir autogestionándose como empresas y mostrar de cara al mercado que son sujetos autosuficientes y capaces de asumir roles que en muchas ocasiones resultan contradictorios.

\subsection{La despolitización de las mujeres}

Antes de empezar a abordar este apartado, debemos partir de una cuestión clave: la obra de Sandberg, al igual que la mayoría de obras del management dirigidas a mujeres, reconocen la necesaria realización de un cambio hacia la igualdad de género dentro de la empresa, en el mundo laboral y en la sociedad en general. Sin embargo, al aproximarnos de forma crítica y desde una mirada feminista al discurso mediante el cual realizan dicha reclamación, vemos que la transformación social que se propone es una transformación individual, que resulta completamente acrítica con el sistema patriarcal y no pretende transformar las estructuras sociales. A continuación, podemos observar cómo la autora declara el descontento con las estructuras desiguales y realiza una llamada a las lectoras para transformar esta realidad:

I believe that if more women lean in, we can change the power structure of our world and expand opportunities for all (Sandberg, 2013: 171).

My hope, of course, is that we won't have to play by these archaic rules forever and that eventually we can all just be ourselves (Sandberg, 2013: 49).

Talking can transform minds, which can transform behaviors, which can transform institutions (Sandberg, 2013: 148).

We can close the leadership gap now (Sandberg, 2013: 171).

I believe women can lead more in the workplace (Sandberg, 2013: 172).

Because the world needs you to change it (Sandberg, 2013: 26).

Major changes can result from these kinds of "nudge techniques," small interventions that encourage people to behave in slightly different ways at critical moments (Sandberg, 2013: 148).

Sin embargo, justo después de señalar que la consecución de la igualdad es vital, se nos advierte de que debemos tener cuidado cuando sacamos a relucir la cuestión de género como variable para la explicación de diferentes problemáticas que se producen tanto en el ámbito del trabajo como en el ámbito social:

At the same time, we must be careful not to inject gender into every discussion (Sandberg, 2013: 150).

Así pues, el descontento con las desigualdades de género que parece hacer explícito la autora y que podría ser fácilmente asociable con una necesidad de articular una 
lucha feminista para la consecución de la igualdad, va de la mano en esta literatura con una despolitización de las mujeres como agentes políticos del feminismo. Dicha realidad parece contradictoria, pero responde a la lógica ambigua del neoliberalismo y a la construcción de las mujeres como sujetos neoliberales que habíamos señalado junto a Scharff. Por esta razón no resulta extraño que en ningún momento se hable de feminismo de forma extendida, y que se prefiera usar el término "igualdad".

Otro rasgo importante es que este proceso de despolitización se nutre del lenguaje de la psicología para conseguir sus objetivos. En este sentido, uno de los primeros pasos discursivos que se llevan a cabo para desarticular la conciencia política de un sujeto feminista es el de situar las barreras y obstáculos derivados de las estructuras patriarcales de la sociedad en el interior de cada mujer:

My argument is that getting rid of these internal barriers is critical to gaining power (Sandberg, 2013: 8).

We internalize the negative messages we get throughout our lives - the messages that say it's wrong to be outspoken, aggressive, more powerful than men (Sandberg, 2013: 8).

We can dismantle the hurdles in ourselves today (Sandberg, 2013: 9).

In addition to the external barriers erected by society, women are hindered by barriers that exist within ourselves (Sandberg, 2013: 8).

A partir del reconocimiento de dichos obstáculos "internos" como los principales causantes de la desigualdad de género, se empieza a articular todo un proceso de individualización de los problemas sociales a hombros de las mujeres. En este sentido, el valor de la confianza se vuelve un valor irrenunciable para transformar la situación de las mujeres. Como vemos, la "solución" que se prescribe por parte de esta obra (que representa de buen grado la literatura popular del management dirigida a mujeres) tiene un carácter completamente psicológico, individual y apolítico:

We hold ourselves back in ways both big and small, by lacking self-confidence, by not raising our hands, and by pulling back when we should be leaning in (Sandberg, 2013: 8).

But I also know that in order to continue to grow and challenge myself, I have to believe in my own abilities (Sandberg, 2013: 38).

I tried to be authentic and shared my truth (Sandberg, 2013: 139).

Esta lógica argumental centrada en explotar las capacidades internas de cada mujer se encuentra directamente asociada al principio de la meritocracia que rige a la sociedad neoliberal y que resulta mucho más opresora cuando se dirige a las mujeres (Littler, 2013; Lewis y Simpson, 2010; Sealy, 2010; Simpson, Ross-Smith y Lewis, 2010). En su caso, la meritocracia sirve como discurso para pasar por alto todos los condicionantes materiales y simbólicos a los que hacen frente cada día las mujeres. Al mismo tiempo, a través de la confianza se pretende impulsar un cambio interno 
que supuestamente va a transformar la situación de desigualdad que perjudica a las mujeres. Por tanto, el cambio que se persigue, se debe internalizar y llevarlo a cabo cada cual en su dimensión individual, y día a día. En esta línea, Sandberg habla de una "revolución interna", una especie de interiorización de la lucha por la igualdad que debe llevar a cada mujer a conseguir mediante acciones individuales cambios globales para el grueso de las mujeres:

We can reignite the revolution by internalizing the revolution. The shift to a more equal world will happen person by person (Sandberg, 2013: 11).

De este modo, se pretende hacer creer al público que la acción individual de cada mujer profesional en base a la consecución de sus propios intereses va a suponer una verdadera transformación social del conjunto de todas las mujeres. Esta lógica psicologicista tiene muchos puntos de confluencia con la literatura de la autoyuda. Así, la autora Vanina Papalini (2013) nos habla de una cultura terapéutica que florece a partir de los años noventa y que prolifera a través de estos escritos que pone en valor un proceso de individualización de los problemas sociales, y se desarrolla en paralelo al apogeo de la creatividad y el discurso de la innovación social como fuente de desarrollo económico. Dichas premisas se articulan para conformar una nueva actitud en el individuo, que intentando huir de la precariedad, apostará por emprender sus propios negocios, y por presentar ideas novedosas que le aseguren un puesto laboral.

Desde una posición crítica, vemos que dicha literatura muestra un total desinterés por realizar una explicación sistémica de los problemas sociales, situando la responsabilidad de la solución en manos individuales, desconectando los lazos que unen entre sí a los diferentes grupos sociales. Un ejemplo de dicha lógica aplicada a la literatura del management en general se puede ver en la obra ¿Quién se ha llevado mi queso? (Johnson 2000), donde se proporcionan claves para concebir los despidos como "una «oportunidad» para pasar a una mejor situación" (Papalini, 2013: 171). Es decir, temas de interés laboral se tratan de forma acrítica perpetuando los mitos y los prejuicios que les rodean (Fernández-Rodríguez y Martínez Lucio, 2014). Así, la literatura de autoayuda tiene un componente de desmovilización social, ya que desconecta a los individuos de la realidad en la que viven. Esto es algo de lo que también se nutre la literatura del management en general y la dirigida a mujeres en particular. Lo podemos ver por ejemplo en la creencia de que el éxito de unas pocas directivas (cargos directivos) puede suponer verdaderamente una mejora en la vida del resto de mujeres a nivel mundial (mujeres que no responden al perfil mainstream de este grupo).

En resumen, si bien existe en Lean In un interés por abordar la cuestión de la igualdad de género en la empresa y en la sociedad, el discurso subyacente a dicha intención es completamente apolítico. La despolitización de las mujeres en la empresa pasa por individualizar problemas sociales como la desigualdad de género, la desigualdad salarial, el acoso laboral, la feminización de ciertas profesiones, los despidos, el paro, o las cuestiones relativas a la conciliación laboral-familiar entre otras. Dicha individualización no persigue más que la destrucción del sujeto político del feminismo, algo que se consigue haciendo creer a las lectoras que son responsables por completo de las barreras materiales y simbólicas que condicionan su desventajosa posición social. Por tanto, si tratásemos de resumir en una sola 
frase el contenido de Lean In, ésta sería la elegida: "Whenever possible, women should substitute "we" for "I" (47), es decir, desarticular el sujeto político del feminismo para que cada cual pase a ocuparse de sus propios problemas. ¡Menuda transformación!

\section{Conclusiones}

A través del análisis de la obra Lean In (Sandberg, 2013), bestseller de la literatura popular del management dirigida a mujeres, se ha podido comprobar cómo el discurso sobre las mujeres profesionales y emprendedoras se sustenta sobre dos lógicas discursivas centrales: la de la autogestión, y la de la negación de la vulnerabilidad y las dependencias, directamente ligadas a la lógica del nuevo espíritu del capitalismo (Boltanski y Chiapello, 2002). Así, se ha podido comprobar que la trampa neoliberal en relación a las mujeres radica en que aquellas cuestiones que antes alejaban a dicho grupo del espacio público - como el cuidado - ahora son puestas en valor y listas para ser rentabilizadas, aumentando la explotación de dicho grupo. De algún modo, la literatura popular del management dirigida a mujeres contribuye a paliar el déficit que la socialización femenina tiene frente al modelo de sujeto neoliberal mediante la reconceptualización de valores como la ambición y el riesgo (Medina-Vicent, 2018b). Así, las características socializadoras de género de las mujeres dejan de suponer un hándicap para el desarrollo de subjetividades instrumentales, y pasan a convertirse en aliadas del sistema neoliberal, realidad que abre un espacio de tensión y de ambivalencias muy complejo para las mujeres.

Irremediablemente, estas operaciones promueven la despolitización del feminismo, ya que solamente mediante la desarticulación del sujeto político del feminismo se puede promover una lógica individualista que aísla a las mujeres y las responsabiliza de su desventajosa posición social en el mundo laboral. Por tanto, se produce aquí una individualización del malestar de las mujeres provocado por la desigualdad de género y de otra índole, que ayuda a su conversión en capital humano listo para su explotación. A su vez, estas operaciones discursivas contribuyen a reconstruir el modelo tradicional del emprendedor, de corte masculino, que ahora se transforma en un sujeto que reconoce sus vínculos de cuidado con otros seres, y carga con la responsabilidad de su gestión.

Como se ha podido observar, esta literatura sitúa los problemas derivados de una estructura patriarcal desigual a hombros de las personas individuales. De este modo, aunque sí existe una apuesta por la consecución de la igualdad de género, en ningún momento se hace referencia a una lucha colectiva para conseguirla, ni tan siquiera a la necesidad de unas políticas sociales que luchen contra estas problemáticas. Tampoco se reflexiona en torno a las posibilidades de articular una política propia dentro de la empresa ni de establecer herramientas para solucionar las desigualdades que se reproducen dentro de dicho ámbito (Pontón y Pastor, 2016). Más bien al contrario, se considera que cada cual debe ser capaz de luchar y solucionar dichos problemas en su día a día. Esta realidad lleva implícita una carga violenta derivada de la racionalidad neoliberal dirigida a mujeres, que les exige una autoproducción en términos de autosuficiencia y supuesta igualdad, y una exigencia de seguir cumpliendo con los roles de género tradicionales. 
En definitiva, a través del análisis de contenido realizado podemos sostener que la literatura popular del management dirigida a mujeres contribuye a la conformación de las mujeres como sujetos neoliberales y emprendedores. Hemos visto que los valores que se subrayan en este tipo de literatura, así como la lógica de la autogestión y la responsabilización individual de los problemas sociales, contribuyen a desarticular el sujeto político del feminismo. Así, convirtiendo a las emprendedoras en mujeres aisladas que consideran que sus éxitos profesionales servirán para una labor de igualdad que repercuta en todas las mujeres, vemos que se va desproveyendo al feminismo de su sentido crítico intrínseco. De este modo, resulta imposible articular un feminismo cosmopolita que permita hacer frente a las exigencias y problemáticas de las mujeres alrededor del mundo (Reverter-Bañón, 2017), y se reproducen las desigualdades de género que ficticiamente estas obras señalan querer destruir.

\section{Referencias bibliográficas}

Alonso, L. E. (2003). La mirada cualitativa en sociología: una aproximación interpretativa. Madrid: Fundamentos.

Alonso, L. E., Fernández Rodríguez, C. J., y Rojo, R. I. (2016). “De la moral del sacrificio a la conciencia de la precariedad. Un análisis cualitativo de los discursos sobre la evolución de la crisis en España”. Política y Sociedad. 53 (2): 353-379.

Alwill, C. (2015). Girl Code: Unlocking Secrets to Success, Sanity, and Happiness for the Female Entrepreneur. New York: Passionista Publishing.

Barry, A., Osbourne, T., y Rose, N. (1996). Foucault and Political Reason: Liberalism, Neoliberalism and Rationalities of Government. London: Routledge.

Boltanski, L., y Chiapello, È. (2002). El nuevo espíritu del capitalismo. Madrid: Akal.

Bourne, K., y Calás, M. (2013). "Becoming 'real' entrepreneurs: Women and the gendered normalization of "work"'. Gender, Work \& Organization. 20 (4): 425-438.

Bröckling, U. (2016). The Entrepreneurial Self: Fabricating a New Type of Subject. London: Sage Publications.

Brown, W. (1995). States of Injury: Power and Freedom in Late Modernity. Princeton: Princeton University Press.

Brown, W. (2003). Neoliberalism and the End of Liberal Democracy. Theory \& Event, 7 (1).

Brown, W. (2016). El pueblo sin atributos. La secreta revolución del neoliberalismo. México: MalPaso.

Bryman, A. (2012). Social Research Methods. Oxford: Oxford University Press.

Chiapello, Ė., y Fairclough, N. (2002). "Understanding the new management ideology: a transdisciplinary contribution from critical discourse analysis and new sociology of capitalism". Discourse \& Society, 13 (2): 185-208.

Clark, T., y Salaman, G. (1996). "The management guru as organizational witchdoctor". Organization. 3 (1): 85-107.

Collins, D. (2000). Management Fads and Buzzwords. Critical-Practical Pespectives. London: Routledge.

Condren, D. (2006). Ambition is Not a Dirty Word. New York: Broadway Books.

Fernández Rodríguez, C. J. (2007). El discurso del management: tiempo y narración. Madrid: Centro de Investigaciones Sociológicas. 
Fernández Rodríguez, C. J. (2017). "Estudios críticos de la gestión: estudios culturales de los conflictos en el mundo del trabajo". Política y Sociedad, 54 (1): 23-44.

Fernández Rodríguez, C. J., y Martín Lucio, M. (2014). "El discurso del despido libre en España: una reflexión sobre el papel de los mitos y los prejuicios en las políticas de empleo españolas". Cuadernos de Relaciones Laborales. 32 (1): 191-219.

Fernández Rodríguez, C. J., y Medina-Vicent, M. (2017). "Los nuevos discursos del management: difusión, impactos y resistencias". Recerca. Revista de pensament i anàlisi. 20: 7-14.

Foucault, M. (2007). Nacimiento de la biopolítica. Buenos Aires: Fondo de Cultura Económica.

Fraser, N. (2014). "De cómo cierto feminismo se convirtió en criada del capitalismo. Y la manera de rectificarlo". Debate Feminista 50. DOI: 131-34. doi:10.1016/S01889478(16)30133-5.

Fraser, N. (2015). Fortunas del feminismo. Madrid: Traficantes de sueños.

Fresier, M. (2014). Raise your hands: a reaction to Lean In (en línea). http://academicarchive.snhu.edu/handle/10474/2484 [consulta 17 de Julio de 2017].

Gantman, E. (2005). Capitalism, Social Privilege and Managerial Ideologies. Aldershot: Ashgate.

Gantman, E. (2017). "En torno al potencial transformador de los CMS (Critical Management Studies)". Recerca. Revista de pensament i anàlisi. 20: 15-33.

Gershon, I. (2011). "Neoliberal agency". Current Antropology. 52 (4): 537-555.

Gill, R. (2014). "Unspeakable inequalities: Post feminism, entrepreneurial subjectivity, and the repudiation of sexism among cultural workers". Social Politics. 21 (4): 509-528.

Gill, R., y Scharff, C. (2011). New Femininities: Postfeminism, Neoliberalism and Subjectivity. Basingstoke: Palgrave Macmillan.

Huczynski, A. (2006). Management Gurus. New York: Routledge.

Huffington, A. (2007). On Becoming Fearless: A Road Map for Women. New York: Brown and Company.

Huffington, A. (2015). Thrive: The Third Metric to Redefining Success and Creating a Life of Well-Being, Wisdom, and Wonder. New York: Harmony Books.

Jackson, B. (2002). "Management gurus: What can we really learn from them?". Mt Eliza Business Review. 4: 67-72.

Jiménez Cano, R. (2014). Facebook y Apple ofrecen congelar los óvulos a sus empleadas (en línea). https://elpais.com/tecnologia/2014/10/15/actualidad/1413333970_087854.html [consulta 17 de Julio de 2017].

Johnson, S. (2000). ¿Quién se ha llevado mi queso? Barcelona: Urano.

Kay, K., y Shipman, C. (2014). The Confidence Code: The Science And Art of Self-Assuman$c E$. New York: Harper Business.

Kelan, E. (2008). "The discursive construction of gender in contemporary management literature". Journal of Business Ethics. 81 (2): 427-445.

Kelan, E. (2009). Performing Gender at Work. London and New York: Palgrave Macmillan.

Kozmetsky, R. (1992). La mujer en los negocios. Barcelona: Ediciones Granica.

Laval C., y Dardot, P. (2013). La nueva razón del mundo. Madrid. Gedisa.

Lewis, P. (2010). “Mumpreneurs': Revealing the Post-feminist Entrepreneur”. En P. Lewis y R. Simpson (Eds.), Revealing and Concealing Gender: Issues of Visibility in Organizations (pp. 124-138). London: Palgrave Macmillan.

Lewis, P. (2012). "Post-Feminism and Entrepreneurship: Interpreting Disgust in a Female Entrepreneurial Narrative". En R. Simpson, N. Slutskaya, P. Lewis, y H. Höpfl (Eds.), Dirty Work: Concepts and Identities (pp. 223-238). London: Palgrave Macmillan. 
Lewis, P., y Simpson, R. (2010). "Meritocracy, difference and choice: women's experiences of advantage and disadvantage at work". Gender in Management: An International Journal. 25 (3): 165-169.

Lischinsky, A. (2008). "Examples as persuasive argument in popular management literature". Discourse \& Communication. 2 (3): 243-269.

Littler, J. (2013). "Meritocracy as Plutocracy: The Marketising of «Equality» Under Neoliberalism". New Formations. 80-81: 52-72.

McNay, L. (2009). "Self as Enterprise Dilemmas of Control and Resistance in Foucault's The Birth of Biopolitics". Theory, Culture \& Society. 26 (6): 55-77.

Medina-Vicent, M. (2018a). "Flirting with Neoliberalism: The Transfiguration of Feminist Political Awareness". NORA. Nordic Journal of Feminist and Gender Research. 28 (1): 1-7. DOI: $10.1080 / 08038740.2018 .1424728$

Medina-Vicent, M. (2018b). Género y management en el marco neoliberal. Un análisis crítico para la emergencia de liderazgos feministas. Castelló de la Plana: Universitat Jaume I.

Mercadé, A. (1998). Mujer emprendedora. Claves para crear y dirigir empresas excelentes. Barcelona: Ediciones Granica.

Mercadé, A. (2007). Dirigir en femenino. Barcelona: Gestión 2000.

Muñoz-Rodríguez, D., y Santos-Ortega, A. (2017). "Las cárceles del capital humano: trabajo $\mathrm{y}$ vidas precarias en la juventud universitaria". Recerca, Revista de pensament $i$ anàlisi. 20: $59-78$.

Papalini, V. (2013). Recetas para sobrevivir a las exigencias del neocapitalismo (en línea). http://nuso.org/articulo/recetas-para-sobrevivir-a-las-exigencias-del-neocapitalismo-o-de-como-la-autoayuda-se-volvio-parte-de-nuestro-sentido-comun/ [consulta 17 de Julio de 2017].

Pateman, C. (1995). El contrato sexual. México DF: Anthropos.

Pérez, S. (2013). Sheryl Sandberg's «Lean In» Book Tops Amazon's Best Sellers List On Its First Day Of Sales (en línea). https://techcrunch.com/2013/03/11/sheryl-sandbergs-lean-in-booktops-amazons-best-sellers-list-on-its-first-day-of-sales/ [consulta 17 de Julio de 2017].

Pongratz, H. J., y Voß, G. G. (2003). "From employee to 'entreployee'”. Concepts and Transformation. 8 (3): 239-254.

Pontón, P. y Pastor, I. (2016). "Los discursos de la igualdad en la empresa. El caso de la industria química de Tarragona”. Cuadernos de Relaciones Laborales. 34 (1): 129-149.

Reverter-Bañón, S. (2003). "La perspectiva de género en la filosofía”. Feminismo/s. 1: 33-50.

Reverter-Bañón, S. (2017). “Cosmopolitismo Feminista contra Globalización”. Araucaria. 19: 301-325.

Rottenberg, C. (2013). “The Rise of Neoliberal Feminism”. Cultural Studies. 28 (3): 418437.

Rottenberg, C. (2014). "Happiness and the liberal imagination: How superwoman became balanced”. Feminist Studies. 40 (1): 144-168.

Rottenberg, C. (2017). "Neoliberal Feminism and the Future of Human Capital". Signs: Journal of Women in Culture and Society. 42 (2): 329-348.

Sandberg, S. (2002). Why we have too few women leaders (en línea). https://www.ted.com/talks/ sheryl_sandberg_why_we_have_too_few_women_leaders [consulta 17 de Julio de 2017].

Sandberg, S. (2013). Lean In. Women, Work and the Will to Lead. New York: Alfred A. Knopf.

Sandberg, S., y Grant, A. (2015). Speaking While Female (en línea). https://www.nytimes. com/2015/01/11/opinion/sunday/speaking-while-female.html [consulta 17 de Julio de 2017]. 
Scharff, C. (2016a). "Gender and Neoliberalism. Young women as ideal neoliberal subjects". En S. Springer, K. Birch, y J. MacLeavy (Eds.), The Handbook of Neoliberalism (pp. 217226). New York: Routledge.

Scharff, C. (2016b). Repudiating Feminism. Young Women in a Neoliberal World. London and New York: Routledge.

Scharff, C. (2016c). "The Psychic Life of Neoliberalism: Mapping the Contours of Entrepreneurial Subjectivity". Theory, Culture \& Society. 33 (6): 107-122.

Sealy, R. (2010). "Changing perceptions of meritocracy in senior women's careers". Gender in Management: An International Journal. 25 (3): 184-197.

Sennett, R. (2000). La corrosión del carácter. Barcelona: Anagrama.

Sennett, R. (2006). La cultura del nuevo capitalismo. Barcelona: Anagrama.

Sennett, R. (2009). El artesano. Barcelona: Anagrama.

Serrano, A., y Martín, P. (2017). “From 'Employab-ility' to 'Entrepreneurial-ity' in Spain: youth in the spotlight in times of crisis". Journal of Youth Studies. 20 (7): 1-24.

Simpson, R., Ross-Smith, A., y Lewis, P. (2010). "Merit, special contribution and choice". Gender in Management: An International Journal. 25 (3): 198-207.

Slaughter, A. M. (2012). Why Women Still Can't Have It All (en línea). https://www.theatlantic.com/magazine/archive/2012/07/why-women-still-cant-have-it-all/309020/ [consulta 17 de Julio de 2017].

Stewart, B. (2013). “Lean In? Run Away!” Thought \& Action. Fall 2013: 151-153.

Taylor, A. (2016). "Sheryl Sandberg and Roxane Gay: The Limits and Possibilities of Contemporary Blockbuster Feminism”. En A. Taylor (ed.), Celebrity and the Feminist Blockbuster (pp. 197-233). London: Palgrave Macmillan.

Wilson, K. (2011). “'Race', Gender and Neoliberalism: changing visual representations in development". Third World Quarterly. 32 (2): 315-331.

Wilson, K. (2012). Race, Racism and Development. London: Zed Books.

Wilson, K. (2015). "Towards a radical re-appropriation: Gender, development and neoliberal feminism”. Development and Change. 46 (4): 803-832. 